\title{
Orbifolds, defects and sphere partition function
}

\author{
Kazuo Hosomichi \\ Department of Physics, National Taiwan University, \\ Taipei 10617, Taiwan \\ Physics Division, National Center for Theoretical Sciences, \\ National Tsing-Hua University, \\ Hsinchu 30013, Taiwan \\ E-mail: hosomiti@phys.ntu.edu.tw
}

ABSTRACT: Gauge theories in the presence of codimension two vortex defects are known to be related to the theories on orbifolds. By using this relation we study the localized path integrals of $2 \mathrm{D} \mathcal{N}=(2,2)$ SUSY gauge theories with point-like vortex defects. We present a formula for the correlation functions of vortex defects inserted at the north and the south poles of squashed spheres. For Abelian gauge theories the correlators are locally constant as functions of the parameters of the defect, but exhibit discontinuity at some threshold values determined from the R-charges of the matter multiplets. For non-Abelian gauge groups the correlators depend non-trivially on the types of gauge symmetry breaking due to the defects.

KeYwords: Supersymmetric gauge theory, Field Theories in Lower Dimensions

ARXiv EPRINT: 1507.07650 


\section{Contents}

1 Introduction 1

2 Rigid SUSY on curved 2D surfaces 3

3 Ellipsoid partition function $\quad 6$

4 Defects 12

5 Concluding remarks 21

\section{Introduction}

In quantum field theories in different dimensions, there are interesting class of operators which are not defined in the ordinary way as functionals of fields. These operators, sometimes called defects, are defined by requiring specific singular behavior on the fields around them. One example is the 't Hooft operators [1] in 4D gauge theories supported along one-dimensional paths, whose definition involves requiring the gauge field to develop Dirac monopole singularity along the paths. Another example is the vortex defects supported on codimension two submanifolds. For 4D gauge theories, they are supported along 2D surfaces and therefore called surface defects. They are defined by the singular behavior of the gauge field $A$

$$
A \sim \eta \cdot d \varphi
$$

near the defect. Here $\eta$ is an element of the Lie algebra, and $\varphi$ is the angular coordinate on the 2D plane transverse to and centered at the defect. See [2] for a review on the recent developments. Similar definition in 3D supersymmetric gauge theories leads to 1D defects which are sometimes called vortex loops.

These defects will allow us to study new aspects of quantum field theories related to monopoles or other solitons carrying topological charges in gauge theories. It is particularly interesting to study such defects in the classes of supersymmetric theories in 2, 3 or 4 dimensions where various dualities or nontrivial relations among observables are known. For example, the inclusion of surface defects in $4 \mathrm{D} \mathcal{N}=2$ supersymmetric gauge theories has been studied in the computation of instanton partition functions [3-12], superconformal indices [13-17] or sphere partition functions [18-21], and the results led to a more detailed understanding of the relation between $4 \mathrm{D} \mathcal{N}=2$ SUSY theories and $2 \mathrm{D}$ conformal field theories [22-24], topological field theories or topological strings. The loop operators in 4D $\mathcal{N}=2$ theories were studied from a similar viewpoint in [25-27]; see [28] for a review. Another interesting point is that some of the defect operators can also be described alternatively by a lower-dimensional field theory on their worldvolume interacting with the fields in the bulk [18, 29]. 
A powerful computational tool to evaluate supersymmetric observables explicitly is the localization principle. To use this for supersymmetric path integrals with defect observables, we need to know how to perform the path integration with modified boundary condition on fields. For 't Hooft loop operators in $4 \mathrm{D} \mathcal{N}=2$ supersymmetric gauge theories this was studied in $[26,30]$, where a result fully consistent with the prediction of S-duality was recovered by a careful localization analysis. Similar analysis was made for vortex loop operators (1.1) in 3D SUSY gauge theories in [31, 32].

The purpose of this paper is to study the codimension two vortex defects of the type (1.1) in 2D $\mathcal{N}=(2,2)$ SUSY gauge theories, which are actually local operators. We study the defects by putting two of them at the north and the south poles of the sphere $S^{2}$ and evaluating their correlation function explicitly. The key idea in our analysis is the equivalence of the gauge theories in the presence of the defect and the gauge theories on orbifolds [33]. This equivalence was relevant in the description of the moduli space of instantons in the surface defect background and the computation of the so-called ramified instanton partition function, see [11] and the references therein. Based on the same idea, a formula for the $S^{4}$ partition function of $\mathcal{N}=2$ SUSY gauge theories in the presence of a surface defect was proposed in [19].

We study the defect correlators by reducing the path integral to a finite-dimensional integral and sum using the idea of Coulomb branch localization. The integrand then depends on the parameter $\eta$ of the vortex defects (1.1). When the gauge group is Abelian, it turns out that the dependence on $\eta$ can be completely absorbed into the redefinition of the remaining integration variables when $\eta$ is sufficiently small. As a function of $\eta$ the defect correlator is therefore locally constant, but there are threshold values of $\eta$ at which the value of correlators jumps. The detail of this behavior is shown in the example of U(1) gauge theory (SQED) with $N_{\mathrm{F}}$ electrons and $N_{\mathrm{A}}$ positrons. For non-Abelian gauge theories, the above discontinuous behavior of the integrand leads to a non-trivial dependence of the defect correlator on the type of gauge symmetry breaking at the poles, characterized by the Levi subgroups. We illustrate this by calculating a few sample defect correlators in the $\mathrm{U}(N)$ SQCD with $N_{\mathrm{F}}$ fundamental and $N_{\mathrm{A}}$ anti-fundamental chiral multiplets.

The organization of this paper is as follows. Section 2 is a review of the construction of $2 \mathrm{D} \mathcal{N}=(2,2)$ SUSY gauge theories on squashed spheres, in which we set up the necessary conventions. We will restrict ourselves to the theories of vector and chiral multiplets. In section 3 we revisit the computation of exact partition functions using the index theorem and the fixed point formula. In section 4 we study the localized path integral on the defect background by making connection with the partition functions on orbifolds of $S^{2}$, and propose a formula for the defect correlators. We conclude with a few remarks in section 5 .

Notations. We use Pauli's matrices as the 2D gamma matrices $\gamma^{1}, \gamma^{2}$ and the chirality matrix $\gamma^{3}$. Their antisymmetrized products are denoted as $\gamma^{a b}=\gamma^{[a} \gamma^{b]}$. The charge conjugation matrix $\epsilon$ is anti-symmetric and satisfies $\epsilon \gamma^{a} \epsilon^{-1}=-\left(\gamma^{a}\right)^{T}$. Explicitly,

$$
\epsilon=\left(\begin{array}{cc}
0 & 1 \\
-1 & 0
\end{array}\right), \quad \gamma^{1}=\left(\begin{array}{ll}
0 & 1 \\
1 & 0
\end{array}\right), \quad \gamma^{2}=\left(\begin{array}{cc}
0 & -i \\
i & 0
\end{array}\right), \quad \gamma^{3}=\left(\begin{array}{cc}
1 & 0 \\
0 & -1
\end{array}\right)
$$


For bilinear products of spinors we use the short-hand notations,

$$
\xi \psi \equiv \xi^{\alpha} \epsilon_{\alpha \beta} \psi^{\beta}, \quad \xi \gamma^{a} \psi \equiv \xi^{\alpha} \epsilon_{\alpha \beta}\left(\gamma^{a}\right)_{\gamma}^{\beta} \psi^{\gamma}, \quad \text { etc. }
$$

Note that $\epsilon_{\alpha \beta}$ is antisymmetric while $\left(\epsilon \gamma^{a}\right)_{\alpha \beta}$ are all symmetric.

\section{Rigid SUSY on curved 2D surfaces}

We review here the construction of a class of $\mathcal{N}=(2,2)$ SUSY gauge theories on curved backgrounds. The results summarized here were obtained by $[34,35]$ for the SUSY gauge theories on round sphere $S^{2}$, and later generalized by [36] for squashed spheres.

Killing spinors. We consider two-dimensional spaces with Killing spinors $\xi, \bar{\xi}$ satisfying

$$
\begin{aligned}
& D_{m} \xi \equiv\left(\partial_{m}+\frac{1}{4} \omega_{m}^{a b} \gamma^{a b}-i V_{m}\right) \xi=\gamma_{m} \xi^{\prime} \\
& D_{m} \bar{\xi} \equiv\left(\partial_{m}+\frac{1}{4} \omega_{m}^{a b} \gamma^{a b}+i V_{m}\right) \bar{\xi}=\gamma_{m} \bar{\xi}^{\prime} \text { for some } \xi^{\prime}, \bar{\xi}^{\prime} .
\end{aligned}
$$

Here $V_{m}$ is the background vector field which gauges the vector $\mathrm{U}(1) \mathrm{R}$-symmetry $\mathrm{R}_{\mathrm{V}}$. So the spinor $\xi$ carries the $\mathrm{R}$-charge $\mathrm{R}_{\mathrm{V}}=+1$ and $\bar{\xi}$ carries $\mathrm{R}_{\mathrm{V}}=-1$. Throughout this paper we take the spinors $\xi, \bar{\xi}$ to be Grassmann even. Most of the time we focus on backgrounds on which the Killing spinors satisfy

$$
D_{m} \xi=\frac{i H}{2} \gamma_{m} \xi, \quad D_{m} \bar{\xi}=\frac{i H}{2} \gamma_{m} \bar{\xi}
$$

where $H$ a scalar auxiliary field.

An example of supersymmetric curved backgrounds is the round sphere $S^{2}$ of radius $\ell$ with the background fields $V_{m}=0, H=1 / \ell$. The vielbein and spin connection are written in polar coordinates as

$$
e^{1}=\ell d \theta, \quad e^{2}=\ell \sin \theta d \varphi, \quad \omega^{12}=-\cos \theta d \varphi .
$$

The Killing spinor equation (2.2) then has a solution

$$
\xi=e^{-\frac{i \varphi}{2}}\left(\begin{array}{r}
i \sin \frac{\theta}{2} \\
\cos \frac{\theta}{2}
\end{array}\right), \quad \bar{\xi}=e^{\frac{i \varphi}{2}}\left(\begin{array}{c}
\cos \frac{\theta}{2} \\
i \sin \frac{\theta}{2}
\end{array}\right) .
$$

As has been shown in [36], one can squash the sphere into an ellipsoid of axis-lengths $\ell, \ell, \tilde{\ell}$ embedded in $\mathbb{R}^{3}$ preserving the supersymmetry corresponding to the above $\xi, \bar{\xi}$. The natural choice of vielbein and the corresponding spin connection are

$$
e^{1}=f(\theta) d \theta, \quad e^{2}=\ell \sin \theta d \varphi, \quad \omega^{12}=-\frac{\ell}{f(\theta)} \cos \theta d \varphi,
$$

with $f(\theta) \equiv \sqrt{\ell^{2} \cos ^{2} \theta+\tilde{\ell}^{2} \sin ^{2} \theta}$. The spinor fields (2.4) satisfy (2.1) if the background fields are chosen as

$$
H=\frac{1}{f}, \quad V=\frac{1}{2}\left(\frac{\ell}{f}-1\right) d \varphi .
$$

In fact, the background defined by (2.5), (2.6) admits the Killing spinors (2.4) for arbitrary choice of the function $f(\theta)$. 
Construction of SUSY gauge theories. In this paper we restrict our attention to the $\mathcal{N}=(2,2)$ supersymmetric gauge theories of vector and chiral multiplets. The vector multiplet consists of the Lie algebra valued fields - the gauge field $A_{m}$, two real scalars $\rho, \sigma$, spinors $\lambda, \bar{\lambda}$ and the auxiliary scalar field $D$. They transform under supersymmetry as

$$
\begin{aligned}
\mathbf{Q} A_{m} & =\frac{1}{2}\left(\xi \gamma_{m} \bar{\lambda}+\bar{\xi} \gamma_{m} \lambda\right), \\
\mathbf{Q} \rho & =\frac{1}{2}\left(\xi \gamma_{3} \bar{\lambda}+\bar{\xi} \gamma_{3} \lambda\right), \\
\mathbf{Q} \sigma & =\frac{i}{2}(\xi \bar{\lambda}-\bar{\xi} \lambda), \\
\mathbf{Q} \lambda & =\frac{1}{2} \gamma^{m n} \xi F_{m n}-\gamma^{3 m} D_{m}(\xi \rho)-i \gamma^{m} D_{m}(\xi \sigma)-\gamma^{3} \xi[\rho, \sigma]+\xi D, \\
\mathbf{Q} \bar{\lambda} & =\frac{1}{2} \gamma^{m n} \bar{\xi} F_{m n}-\gamma^{3 m} D_{m}(\bar{\xi} \rho)+i \gamma^{m} D_{m}(\bar{\xi} \sigma)+\gamma^{3} \xi[\rho, \sigma]-\bar{\xi} D, \\
\mathbf{Q} D & =\frac{1}{2} D_{m}\left(\xi \gamma^{m} \bar{\lambda}-\bar{\xi} \gamma^{m} \lambda\right)-\frac{i}{2}\left[\rho, \xi \gamma_{3} \bar{\lambda}-\bar{\xi} \gamma_{3} \lambda\right]+\frac{1}{2}[\sigma, \xi \bar{\lambda}-\bar{\xi} \lambda] .
\end{aligned}
$$

A chiral multiplet consists of a scalar $\phi$, a spinor $\psi$ and an auxiliary scalar $F$ in a complex representation $\Lambda$ of the gauge group. The multiplet is labeled by the vector R-charge of the lowest component,

$$
\mathrm{R}_{\mathrm{V}}[\phi]=2 q .
$$

The conjugate fields $\bar{\phi}, \bar{\psi}, \bar{F}$ form an anti-chiral multiplet in the representation $\bar{\Lambda}$ of the gauge group. The supersymmetry acts on the fields as

$$
\begin{aligned}
& \mathbf{Q} \phi=\xi \psi \\
& \mathbf{Q} \bar{\phi}=\bar{\xi} \bar{\psi} \\
& \mathbf{Q} \psi=-\gamma^{m} \bar{\xi} D_{m} \phi+i \gamma^{3} \bar{\xi} \rho \phi-\bar{\xi} \sigma \phi-q \cdot \phi \gamma^{m} D_{m} \bar{\xi}+\xi F \\
& \mathbf{Q} \bar{\psi}=-\gamma^{m} \xi D_{m} \bar{\phi}-i \gamma^{3} \xi \bar{\phi} \rho-\xi \bar{\phi} \sigma-q \cdot \bar{\phi} \gamma^{m} D_{m} \xi-\bar{\xi} \bar{F} \\
& \mathbf{Q} F=-\bar{\xi}\left(\gamma^{m} D_{m} \psi-i \gamma^{3} \rho \psi-\sigma \psi-i \bar{\lambda} \phi\right)-q \cdot D_{m} \bar{\xi} \gamma^{m} \psi \\
& \mathbf{Q} \bar{F}=+\bar{\xi}\left(\gamma^{m} D_{m} \bar{\psi}+i \gamma^{3} \bar{\psi} \rho-\bar{\psi} \sigma+i \bar{\phi} \lambda\right)+q \cdot D_{m} \xi \gamma^{m} \bar{\psi}
\end{aligned}
$$

Here the fields belonging to the representation $\Lambda(\bar{\Lambda})$ of the gauge group is regarded as column vectors (resp. row vectors), on which the vector multiplet fields act from the left (resp. right).

The supersymmetry $\mathbf{Q}$ squares into a sum of bosonic symmetries,

$$
\begin{aligned}
\mathbf{Q}^{2} & =\operatorname{Lie}(v)+\operatorname{Lorentz}\left(\theta_{a b}\right)+\operatorname{Weyl}(\omega)+\operatorname{Gauge}(\Sigma)+\mathrm{R}_{\mathrm{V}}(\alpha)+\mathrm{R}_{\mathrm{A}}(\beta), \\
\text { (example) } \quad \mathbf{Q}^{2} \lambda & =v^{m} \partial_{m} \lambda+\frac{1}{4} \theta_{a b} \gamma^{a b} \lambda+\frac{3}{2} \omega \lambda+[\Sigma, \lambda]+\alpha \lambda+\beta \gamma_{3} \lambda,
\end{aligned}
$$

with the transformation parameters $v^{m}=-\bar{\xi} \gamma^{m} \xi$ and

$$
\begin{aligned}
\theta_{a b} & =e_{a}^{m} e_{b}^{n} D_{[m} v_{n]}+v^{m} \omega_{m, a b}, \\
\omega & =\frac{1}{2} D_{m} v^{m}
\end{aligned}
$$




\begin{tabular}{|c|cc|ccccc|cccccc|}
\hline fields & $\xi$ & $\bar{\xi}$ & $A_{m}$ & $\rho \pm i \sigma$ & $\lambda$ & $\bar{\lambda}$ & $D$ & $\phi$ & $\bar{\phi}$ & $\psi$ & $\bar{\psi}$ & $F$ & $\bar{F}$ \\
\hline scale & $-\frac{1}{2}$ & $-\frac{1}{2}$ & 0 & 1 & $\frac{3}{2}$ & $\frac{3}{2}$ & 2 & $q$ & $q$ & $q+\frac{1}{2}$ & $q+\frac{1}{2}$ & $q+1$ & $q+1$ \\
\hline $\mathrm{R}_{\mathrm{V}}$ & 1 & -1 & 0 & 0 & 1 & -1 & 0 & $2 q$ & $-2 q$ & $2 q-1$ & $1-2 q$ & $2 q-2$ & $2-2 q$ \\
\hline $\mathrm{R}_{\mathrm{A}}$ & $\gamma_{3}$ & $-\gamma_{3}$ & 0 & \pm 2 & $\gamma_{3}$ & $-\gamma_{3}$ & 0 & 0 & 0 & $\gamma_{3}$ & $-\gamma_{3}$ & 0 & 0 \\
\hline
\end{tabular}

Table 1. the scaling weight and the R-charge of the fields.

$$
\begin{aligned}
\Sigma & =\bar{\xi} \xi \sigma+i \bar{\xi} \gamma_{3} \xi \rho-i v^{m} A_{m} \\
\alpha & =\frac{1}{4}\left(\bar{\xi} \gamma^{m} D_{m} \xi-D_{m} \bar{\xi} \gamma^{m} \xi\right)-i v^{m} V_{m} \\
\beta & =\frac{1}{4}\left(\bar{\xi} \gamma^{m 3} D_{m} \xi-D_{m} \bar{\xi} \gamma^{m 3} \xi\right) .
\end{aligned}
$$

Note that the transformation rules are invariant under Weyl rescalings which transform the metric as a weight -2 object, $\tilde{g}_{m n}=g_{m n} e^{2 \omega}$. The scaling weights, $\mathrm{R}_{\mathrm{V}}$ and the $\mathrm{R}_{\mathrm{A}}$-charges of the fields are summarized in the table 1.

As the kinetic terms for these fields one can take

$$
\begin{aligned}
\mathcal{L}_{\mathrm{vec}}= & \operatorname{Tr}\left[\left(F_{12}-H \rho\right)^{2}+(D+H \sigma)^{2}+D_{m} \rho D^{m} \rho+D_{m} \sigma D^{m} \sigma-[\rho, \sigma]^{2}\right. \\
& \left.-\bar{\lambda}\left(\gamma^{m} D_{m} \lambda-i \gamma^{3}[\rho, \lambda]-[\sigma, \lambda]\right)\right] \\
\mathcal{L}_{\text {mat }}= & D_{m} \bar{\phi} D^{m} \phi+\bar{\phi}\left\{\rho^{2}+\sigma^{2}+i q H \sigma+\frac{1}{2} q R-q^{2} H^{2}+i D\right\} \phi+\bar{F} F \\
& -\bar{\psi}\left(\gamma^{m} D_{m}-i \gamma^{3} \rho-\sigma-i q H\right) \psi+i \bar{\psi} \bar{\lambda} \phi-i \bar{\phi} \lambda \psi .
\end{aligned}
$$

These are invariant under the SUSY generated by the Killing spinors satisfying the stronger condition (2.2). They are in fact SUSY exact,

$$
\begin{aligned}
\mathcal{L}_{\mathrm{vec}} & =\mathbf{Q}_{\xi} \mathbf{Q}_{\bar{\xi}} \operatorname{Tr}\left[\bar{\lambda} \lambda+4 i \sigma D+2 i H \sigma^{2}\right], \\
\mathcal{L}_{\text {mat }} & =\mathbf{Q}_{\xi} \mathbf{Q}_{\bar{\xi}}[-\bar{\psi} \psi-2 \bar{\phi} \sigma \phi-i(2 q-1) H \bar{\phi} \phi],
\end{aligned}
$$

so they play the role of regulators. Namely, since the values of SUSY path integrals do not depend on the coefficients multiplying them, one can make them large so that the saddle point approximation becomes exact and the path integral localizes onto the moduli space of Q-invariant configurations. On the other hand, the FI-theta term for Abelian vector multiplet,

$$
\mathcal{L}_{\mathrm{FI}}=-i \zeta D+\frac{i \theta}{2 \pi} F_{12},
$$

is SUSY invariant but not exact, so the expectation values of supersymmetric observables depend non-trivially on the couplings $\zeta, \theta$. Another coupling is the mass for chiral multiplets, which can be introduced by coupling some external vector multiplets to the matter flavor symmetries and turning on its scalar components, as we will see later. 


\section{$3 \quad$ Ellipsoid partition function}

The exact formula for partition functions on the ellipsoid (2.5) has been derived by [36], generalizing the earlier result for the round sphere [34,35]. We revisit here the derivation of the formula using the index theorem.

Using Coulomb branch localization, the path integral can be shown to localize onto the saddle point configurations which minimize the bosonic part of $\mathcal{L}_{\text {vec }}$. The saddle points are labeled by the parameters $s, a$ taking values in the Cartan subalgebra. Up to gauge choices, the bosonic fields in vector multiplet take the following form

$$
\sigma=\frac{a}{\ell}, \quad D=-\frac{a}{f \ell}, \quad A=s \cdot \cos \theta d \varphi, \quad \rho=-\frac{s}{\ell} .
$$

while the matter fields all vanish. The parameter $s$ is the magnetic flux through the ellipsoid, so it is subject to GNO quantization. The path integral thus decomposes into two steps: one first integrates over the fluctuations around each saddle point under Gaussian approximation and obtain the so-called one-loop determinant. The result is then summed (integrated) over all the saddle points.

From determinant to character. The one-loop determinant can be most easily computed by moving to a new set of path integration variables. Let us explain this change of variables for the chiral multiplet first. For simplicity we consider a chiral multiplet with unit charge under a $\mathrm{U}(1)$ vector multiplet which take the saddle point value (3.1) with $a \in \mathbb{R}, s \in \mathbb{Z} / 2$. From the fields $\phi, \psi, F$ we define a boson $\mathbf{X}$, a fermion $\boldsymbol{\Xi}$ and their superpartners $\mathbf{Q X}, \mathbf{Q} \boldsymbol{\Xi}$ as follows,

$$
\begin{array}{llll}
\mathbf{X} \equiv \phi, & \mathbf{Q X} \equiv \xi \psi, & \boldsymbol{\Xi} \equiv \bar{\xi} \psi, & \mathbf{Q} \boldsymbol{\Xi}=F-\bar{\xi} \gamma^{m} \bar{\xi} D_{m} \phi+i \bar{\xi} \gamma^{3} \bar{\xi} \rho \phi \\
\overline{\mathbf{X}} \equiv \bar{\phi}, & \mathbf{Q X} \equiv \overline{\boldsymbol{X}} \bar{\psi}, & \mathbf{\Xi} \equiv \xi \bar{\psi}, & \mathbf{Q} \overline{\boldsymbol{\Xi}}=\bar{F}-\xi \gamma^{m} \xi D_{m} \bar{\phi}-i \xi \gamma^{3} \xi \bar{\phi} \rho .
\end{array}
$$

Note that $\mathbf{X}$ is Grassmann-even while $\boldsymbol{\Xi}$ is odd, but they are both Lorentz scalars. One can show that the relation between $\phi, \psi, F$ and the new fields $\mathbf{X}, \mathbf{Q X}, \boldsymbol{\Xi}, \mathbf{Q} \boldsymbol{\Xi}$ is local and invertible. The path integral over the fluctuations then gives rise to the ratio of determinants of $\mathbf{Q}^{2}$ acting on the fields $\mathbf{X}$ and $\boldsymbol{\Xi}$,

$$
Z_{1-\text { loop }}=\frac{\left.\operatorname{det} \mathbf{Q}^{2}\right|_{\Xi}}{\left.\operatorname{det} \mathbf{Q}^{2}\right|_{\mathbf{X}}}
$$

This can be understood by thinking of the $\mathbf{Q}$-exact path integration weight $e^{-S_{\text {reg }}}=$ $e^{-\mathbf{Q} \mathcal{V}}$ with

$$
\begin{aligned}
\mathcal{V} & =\int d^{2} x \sqrt{g}(\overline{\mathbf{X}} \cdot \mathbf{Q} \mathbf{X}+\overline{\boldsymbol{\Xi}} \cdot \mathbf{Q} \boldsymbol{\Xi}), \\
S_{\mathrm{reg}} & =\int d^{2} x \sqrt{g}\left(\mathbf{Q} \overline{\mathbf{X}} \cdot \mathbf{Q X}+\overline{\mathbf{X}} \cdot \mathbf{Q}^{2} \mathbf{X}+\mathbf{Q} \overline{\boldsymbol{\Xi}} \cdot \mathbf{Q} \boldsymbol{\Xi}-\overline{\boldsymbol{\Xi}} \cdot \mathbf{Q}^{2} \boldsymbol{\Xi}\right) .
\end{aligned}
$$

The above choice of $S_{\text {reg }}$ is the simplest, but does not necessarily have a positive definite bosonic part, so the Wick rotation of some integration variables will be needed. More careful analysis with an $S_{\text {reg }}$ with manifestly positive definite bosonic part should lead to the same one-loop determinant. 
As a useful intermediate quantity for computing the one-loop determinant (3.3), let us introduce the character

$$
\chi=\operatorname{Tr}_{\mathbf{X}}\left(e^{t \mathbf{Q}^{2}}\right)-\operatorname{Tr} \Xi\left(e^{t \mathbf{Q}^{2}}\right) .
$$

The fields $\mathbf{X}$ and $\boldsymbol{\Xi}$ are scalars with the vector R-charge $2 q$ and $2 q-2$. The square of the SUSY acts on scalar fields in general as

$$
\mathbf{Q}^{2}=\frac{1}{\ell}\left\{i J_{3}+\frac{i}{2} \mathrm{R}_{\mathrm{V}}+\operatorname{Gauge}(a)\right\}, \quad J_{3} \equiv-i \partial_{\varphi} .
$$

The character (3.5) can be computed as an index if there is a differential operator which commutes with $\mathbf{Q}^{2}$ and maps between $\mathbf{X}$ and $\boldsymbol{\Xi}$. Such operators $J^{ \pm}$with the vector Rcharge $\mp 2$ can be constructed using Killing spinors,

$$
J^{+}=\ell\left(\bar{\xi} \gamma^{m} \bar{\xi} D_{m}-i \bar{\xi} \gamma^{3} \bar{\xi} \rho\right), \quad J^{-}=\ell\left(\xi \gamma^{m} \xi D_{m}-i \xi \gamma^{3} \xi \rho\right) .
$$

More explicitly in components,

$$
J^{ \pm}=e^{ \pm i \varphi}\left\{ \pm \frac{\ell}{f} \partial_{\theta}+i \cot \theta \partial_{\varphi}+\frac{s}{\sin \theta}+\frac{1}{2} \mathrm{R}_{\mathrm{V}}\left(\frac{\ell}{f}-1\right) \cot \theta\right\} .
$$

Note that when $f(\theta)=\ell$ these operators coincide with the angular momentum operators in the background magnetic flux $s$, justifying the name $J^{ \pm}$. Therefore, on the round sphere the character can be computed by expanding the fields $\mathbf{X}$ and $\boldsymbol{\Xi}$ into monopole harmonics [37].

Monopole harmonics is a convenient basis to expand the fields on $S^{2}$ coupled to the background monopole gauge field

$$
A=s \cos \theta d \varphi, \quad F=-s \sin \theta d \theta d \varphi=-\frac{s}{2 r^{3}} \epsilon_{a b c} x_{a} d x_{b} d x_{c}, \quad\left(r \equiv \sqrt{x_{a} x_{a}}\right)
$$

where $x_{a}$ is the Cartesian coordinate of the embedding space $\mathbb{R}^{3}$. The angular momentum operators for a particle with unit electric charge is

$$
\begin{aligned}
& J_{a}^{(s)}=-i \epsilon_{a b c} x_{b}\left(\partial_{c}-i A_{c}\right)+\frac{s x_{a}}{r} \\
& J_{3}^{(s)}=-i \partial_{\varphi}, \quad J_{ \pm}^{(s)}=e^{ \pm i \varphi}\left( \pm \partial_{\theta}+i \cot \theta \partial_{\varphi}+\frac{s}{\sin \theta}\right) .
\end{aligned}
$$

Note that $J_{ \pm}^{(s)}$ agree with the differential operators (3.8) which commute with the square of SUSY when the background sphere is round. The monopole harmonics $Y_{j m}^{s}$ transform as the spin- $j$ representation under the action of $J_{a}^{(s)}$. Let us also introduce

$$
D_{ \pm}^{(s)} \equiv \partial_{\theta} \mp \frac{i}{\sin \theta}\left(\partial_{\varphi}-i s \cos \theta\right) .
$$

One can show that the massless Dirac operator for the charged Weyl spinors of \pm chirality on sphere is $D_{\mp}^{(s \pm 1 / 2)}$, where the $\pm 1 / 2$ shift is due to spin connection. Note also that the Hermite conjugate of $D_{ \pm}^{(s)}$ is $D_{\mp}^{(s \pm 1)}$, since the volume element of the unit sphere is $\sin \theta d \theta d \varphi$. It now follows from the important identities

$$
\begin{aligned}
D_{ \pm}^{(s)} J_{a}^{(s)} & =J_{a}^{(s \pm 1)} D_{ \pm}^{(s)}, \\
D_{ \pm}^{(s \mp 1)} D_{\mp}^{(s)} & =s(s \mp 1)-J_{a}^{(s)} J_{a}^{(s)},
\end{aligned}
$$


that $Y_{j m}^{s}$ exists only for $j \in|s|+\mathbb{Z}_{\geq 0}$. One can normalize the monopole harmonics so that the following holds,

$$
D_{ \pm}^{(s)} Y_{j m}^{s}= \pm \sqrt{(j \mp s)(j \pm s+1)} Y_{j m}^{(s \pm 1)} .
$$

The character on the round sphere can be easily evaluated by recalling that the kernel of $J^{+}$(or $J^{-}$) are spanned by the monopole harmonics $Y_{j m}^{s}$ with $m=j$ (resp. $m=-j$ ), and that the allowed values of $j$ are bounded by $|s|$.

$$
\begin{aligned}
\chi(a, s) & =\left.\operatorname{Tr}_{\mathbf{X}}\left(e^{t \mathbf{Q}^{2}}\right)\right|_{\operatorname{Ker} J^{+}}-\left.\operatorname{Tr}_{\Xi}\left(e^{t \mathbf{Q}^{2}}\right)\right|_{\text {Ker } J^{-}} \\
& =\sum_{n \geq 0}\left(x^{n+|s|-i a+q}-x^{-n-|s|-i a+q-1}\right), \quad x \equiv e^{\frac{i t}{\ell}} .
\end{aligned}
$$

This is for the chiral multiplet with unit charge under a $U(1)$ vector multiplet, and the generalization is straightforward. For chiral multiplet in a representation $\Lambda$ of the gauge group, the character at the saddle point $(a, s)$ is given by the sum over the weight vectors $w \in \Lambda$,

$$
\chi_{\Lambda, q}(a, s)=\sum_{w \in \Lambda} \sum_{n \geq 0}\left(x^{n+|s \cdot w|-i a \cdot w+q}-x^{-n-|s \cdot w|-i a \cdot w+q-1}\right) .
$$

Vector multiplet and gauge fixing. Let us turn to the vector multiplet. A convenient set of fields is

$$
\begin{aligned}
\mathbf{X}^{+} & \equiv \bar{\xi} \gamma^{m} \bar{\xi} A_{m}+\bar{\xi} \gamma^{3} \bar{\xi} \rho, & \mathbf{Q X} & =-\frac{1}{2} \bar{\xi} \bar{\lambda} \\
\mathbf{X}^{0} & \equiv \bar{\xi} \gamma^{m} \xi A_{m}+\bar{\xi} \gamma^{3} \xi \rho, & \mathbf{Q X} & =\frac{1}{2} \bar{\xi} \lambda-\frac{1}{2} \xi \bar{\lambda}, \\
\mathbf{X}^{-} & \equiv \xi \gamma^{m} \xi A_{m}+\xi \gamma^{3} \xi \rho, & \mathbf{Q X} & =\frac{1}{2} \xi \lambda, \\
\boldsymbol{\Xi} & \equiv \frac{1}{2} \bar{\xi} \lambda+\frac{1}{2} \xi \bar{\lambda}, & \mathbf{Q} \boldsymbol{\Xi} & =D+\frac{1}{f} \sigma+i \bar{\xi} \gamma^{3} \xi\left(F_{12}-\frac{\rho}{f}\right), \\
\Sigma & \equiv \bar{\xi} \xi \sigma+i \bar{\xi} \gamma^{3} \xi \rho-i v^{m} A_{m}, & \mathbf{Q} \Sigma & =0 .
\end{aligned}
$$

This consists of five Grassmann-even and four Grassmann-odd fields, and the change of variables from $\left(A_{m}, \rho, \sigma, \lambda, \bar{\lambda}, D\right)$ to these fields is local and invertible. In addition, for the gauge fixing we need to introduce the ghost fields $c, \bar{c}, B$ and the BRST symmetry $\mathbf{Q}_{\mathrm{B}}$. The BRST charge $\mathbf{Q}_{\mathrm{B}}$ acts in the standard way on all the physical fields, namely as Gauge $(c)$. For ghost fields, we follow [38] and set the transformation rule on the saddle point $(a, s)$ as

$$
\begin{aligned}
\mathbf{Q}_{\mathrm{B}} c & =c c, & \mathbf{Q} c & =\langle\Sigma\rangle-\Sigma, \\
\mathbf{Q}_{\mathrm{B}} \bar{c} & =B, & \mathbf{Q} \bar{c} & =0, \\
\mathbf{Q}_{\mathrm{B}} B & =0, & \mathbf{Q} B & =v^{m} \partial_{m} \bar{c}+[\langle\Sigma\rangle, \bar{c}] . \quad(\langle\Sigma\rangle=a / \ell)
\end{aligned}
$$

The total supersymmetry $\hat{\mathbf{Q}}=\mathbf{Q}+\mathbf{Q}_{\mathrm{B}}$ then satisfies

$$
\left(\mathbf{Q}+\mathbf{Q}_{\mathrm{B}}\right)^{2}=\frac{1}{\ell}\left\{i J_{3}+\frac{i}{2} \mathrm{R}_{\mathrm{V}}+\operatorname{Gauge}(a)\right\} .
$$


It is important that the constant modes along the direction of Cartan subalgebra should be excluded from the ghost fields $(c, \bar{c}, B)$, since it is the direction along the saddle point locus and should not be gauge fixed.

The character for the vector multiplet can be most easily evaluated by taking as the independent variables the three scalar bosons $\left(\mathbf{X}^{+}, \mathbf{X}^{0}, \mathbf{X}^{-}\right)$, three scalar fermions $(\boldsymbol{\Xi}, c, \bar{c})$ and their $\hat{\mathbf{Q}}$-superpartners. Notice that $\mathbf{X}^{\mp}$ has $\mathbf{R}_{\mathrm{V}}= \pm 2$ while all other fields have $\mathbf{R}_{\mathrm{V}}=0$. The character is therefore the same as that for the adjoint chiral multiplet with $q=1$.

Fixed point formula. The character can also be computed by using the Atiyah-Bott fixed point formula, and this technique can be applied also to the theory on squashed spheres. In this way one can also show that the partition function does not depend on the detail of the squashing. To understand this technique, recall that the character is the difference of traces, where the operator of interest $e^{t \mathbf{Q}^{2}}$ involves a finite rotation $\varphi \rightarrow \varphi+t / \ell$. The trace of such an operator can be expressed as a sum of contributions from the fixed points, the north and the south poles. Let $z \equiv \tan \frac{\theta}{2} e^{i \varphi}$ be the local complex coordinate near the north pole, which is transformed by $e^{t \mathbf{Q}^{2}}$ as

$$
z \rightarrow \tilde{z}=z x . \quad\left(x \equiv e^{i t / \ell}\right)
$$

The north pole contribution to the trace (= diagonal sum) of the operator $e^{t \mathbf{Q}^{2}}$ is then given by the integral of $\delta^{2}(z-\tilde{z})$ multiplied by its value at the north pole. It should be evaluated in a gauge in which there is no Dirac string singularity at the north pole, which is related to (3.9) by the gauge transformation $e^{-i s \varphi}$. The differential operators $J_{[\mathrm{N}]}^{a} \equiv e^{-i s \varphi} J^{a} e^{i s \varphi}$ in this gauge behave near the north pole as,

$$
J_{[\mathrm{N}]}^{+} \simeq \frac{\partial}{\partial \bar{z}}, \quad J_{[\mathrm{N}]}^{-} \simeq-\frac{\partial}{\partial z}, \quad J_{[\mathrm{N}]}^{3}=z \frac{\partial}{\partial z}-\bar{z} \frac{\partial}{\partial \bar{z}}+s .
$$

The north pole contribution to the character (for the chiral multiplet with unit U(1) charge) is then

$$
\left.\chi\right|_{\mathrm{N}}=\left(x^{s-i a+q}-x^{s-i a+q-1}\right) \cdot \int d^{2} z \delta^{2}(z-x z)=\frac{x^{s-i a+q}}{1-x} .
$$

Note that, depending on how to expand into series in $x$, this result can be interpreted as either the contribution of the $J^{+}$-zeromodes of $\mathbf{X}$ or the $J^{-}$-zeromodes of $\boldsymbol{\Xi}$. The south pole contribution can be evaluated in the same way: the differential operators $J_{[\mathrm{s}]}^{a} \equiv e^{i s \varphi} J^{a} e^{-i s \varphi}$ behave around there as

$$
J_{[\mathrm{s}]}^{+} \simeq-\frac{\partial}{\partial w}, \quad J_{[\mathrm{s}]}^{-} \simeq \frac{\partial}{\partial \bar{w}}, \quad J_{[\mathrm{s}]}^{3}=-w \frac{\partial}{\partial w}+\bar{w} \frac{\partial}{\partial \bar{w}}-s \quad\left(w \equiv \cot \frac{\theta}{2} e^{-i \varphi}\right) .
$$

which leads to

$$
\left.\chi\right|_{\mathrm{S}}=\frac{x^{-s-i a+q}}{1-x} .
$$

Combining the two pole contributions expanded into opposite series in $x$ we obtain the character,

$$
\chi=\sum_{n \geq 0}\left(x^{n+s-i a+q}-x^{-1-n-s-i a+q}\right) .
$$


It is invariant under $s \rightarrow-s$, since $s$ is half-integer valued. Note that each term in the infinite sum corresponds to an approximate zeromode of $J^{+}$or $J^{-}$, namely a local holomorphic function,

$$
\mathbf{X}=z^{n}, \quad \boldsymbol{\Xi}=w^{n}, \quad n \in \mathbb{Z}_{\geq 0}
$$

near one of the poles. Note also that the derivation of the character does not depend on the detailed form of the squashed metric, as long as it preserves the $U(1)$ isometry and is regular at the poles.

One-loop determinant and an anomaly. The one-loop determinant is evaluated as the product of the eigenvalues of $\mathbf{Q}^{2}$. For the unit $\mathrm{U}(1)$-charge chiral multiplet, the character (3.24) leads to the determinant

$$
Z_{1 \text {-loop }}=\prod_{n \geq 0} \frac{-n-1-s-i a+q}{n+s-i a+q}=(\text { phase }) \cdot \frac{\Gamma(s-i a+q)}{\Gamma(s+i a+1-q)},
$$

where we sign-flipped all the eigenvalues in the enumerator of the left hand side of the second equality. To keep the invariance of the determinant under $s \rightarrow-s$, one needs an $s$-dependent phase on the right hand side. However, this symmetry is actually anomalous in the path integral, and the formula

$$
Z_{1-\text { loop }}(s, a, q)=\frac{\Gamma(s-i a+q)}{\Gamma(s+i a+1-q)}=Z_{1-\text { loop }}(-s, a, q)(-1)^{2 s},
$$

gives the correct one-loop determinant which leads to the nice factorization property of sphere partition function [34, 35].

To elaborate on this anomaly, let us look into the path integral with respect to the fermion in the chiral multiplet, which is

$$
\int D \bar{\psi} D \psi \exp \left(-\int d^{2} x \sqrt{g} \mathcal{L}\right), \quad \mathcal{L} \equiv-\bar{\psi}\left\{\gamma^{m} D_{m}-i \gamma^{3} \rho-\sigma-\frac{i q}{\ell}\right\} \psi,
$$

with the vector multiplet fields fixed at the saddle point value (3.9). We notice that the squashed sphere geometry (2.5) is invariant under the antipodal map

$$
(\theta, \varphi) \rightarrow(\pi-\theta, \pi+\varphi)
$$

if $f(\theta)=f(\pi-\theta)$. The Lagrangian $\mathcal{L}$ is also invariant if the antipodal map is defined to act on fields of various spins as,

$$
\begin{aligned}
& \text { scalar : } & \tilde{\phi}(\theta, \varphi) & =\phi(\pi-\theta, \varphi+\pi), \\
& \text { spinor : } & \tilde{\psi}(\theta, \varphi) & =i \gamma^{2} \psi(\pi-\theta, \varphi+\pi), \\
\text { vector : } & & \left(\tilde{A}_{\theta}, \tilde{A}_{\varphi}\right)(\theta, \varphi) & =\left(-A_{\theta}, A_{\varphi}\right)(\pi-\theta, \varphi+\pi) .
\end{aligned}
$$

One can easily check that the Killing spinors (2.4) are invariant under this map, and the saddle point configuration (3.1) labeled by $(s, a)$ is transformed to another saddle point with $s$ sign-flipped. This symmetry would lead to the invariance of $Z_{1 \text {-loop }}$ under $s \rightarrow-s$ 
if there were no anomaly. The sign factor $(-1)^{2 s}$ should arise from the non-invariance of the measure.

To see the anomaly explicitly, let us expand the fermions $\psi=\left(\psi^{+}, \psi^{-}\right)$into the eigenfunctions of chiral Dirac operator $D_{ \pm}^{(s)}$ squared,

$$
\psi^{ \pm}(\theta, \varphi)=\sum_{i} \psi_{i}^{ \pm} Y_{i}^{s, \pm}(\theta, \varphi), \quad D_{ \pm}^{(s)} D_{\mp}^{(s)} Y_{i}^{s, \pm}=-\lambda_{i} Y_{i}^{s, \pm}
$$

Let $n_{ \pm}$be the numbers of zeromodes for the chiral (antichiral) components $\psi^{ \pm}$. The integration measure for $\psi$ then takes the form

$$
D \psi \equiv \prod_{\lambda_{i}=0} d \psi_{i}^{+} \cdot \prod_{\lambda_{i}=0} d \psi_{i}^{-} \cdot \prod_{\lambda_{i} \neq 0} d \psi_{i}^{+} d \psi_{i}^{-}
$$

where the three factors correspond to $n_{+}$zeromodes of $\psi^{+}, n_{-}$zeromodes of $\psi^{-}$and the rest (nonzero modes). Note that $n_{+}-n_{-}=-2 s$ from the index theorem. The fermions $\bar{\psi}^{\alpha}$ are expanded into the eigenfunctions $Y_{i}^{-s, \pm}$ in the same way.

The eigenfunctions $Y_{i}^{s,+}, Y_{i}^{s,-}$ of the same nonzero eigenvalue $\lambda_{i}$ are paired by the action of $D_{ \pm}^{(s)}$. They obey the periodicity

$$
Y_{i}^{s, \pm}(\theta, \varphi+2 \pi)=(-1)^{2 s+1} Y_{i}^{s, \pm}(\theta, \varphi)
$$

if the background spin connection and the vector multiplet fields are in the gauge (2.5), (3.1). Also, the antipodal map acts on the chiral Dirac operators as $D_{ \pm}^{(s)} \rightarrow-D_{\mp}^{(-s)}$. Combining all these one can show that the set of eigenfunctions can be chosen to satisfy

$$
\begin{array}{ll}
2 s=\text { even }: & Y_{i}^{s, \pm}(\pi-\theta, \pi+\varphi)=i Y_{i}^{-s, \mp}(\theta, \varphi), \\
2 s=\text { odd }: & Y_{i}^{s, \pm}(\pi-\theta, \pi+\varphi)=Y_{i}^{-s, \mp}(\theta, \varphi) .
\end{array}
$$

The antipodal map transforms $\psi$ to $\tilde{\psi}$ as in (3.29). Their modes $\tilde{\psi}_{i}^{ \pm}$are defined and related to the modes of the original fermion $\psi_{i}^{ \pm}$as follows,

$$
\tilde{\psi}^{ \pm}(\theta, \varphi)=\sum_{i} \tilde{\psi}_{i}^{ \pm} Y_{i}^{-s, \pm}(\theta, \varphi), \quad \tilde{\psi}_{i}^{ \pm}=\left\{\begin{array}{ll} 
\pm i \psi_{i}^{\mp} & (2 s=\text { even }) \\
\pm \psi_{i}^{\mp} & (2 s=\text { odd })
\end{array} .\right.
$$

This leads to the anomaly

$$
D \tilde{\bar{\psi}} D \tilde{\psi}=(-1)^{2 s} D \bar{\psi} D \psi
$$

as claimed, where the sign factor arises from the zeromode part of the measure. Let us note here that one can work out the full eigenfunctions and eigenmodes explicitly for the round sphere, since in that case the operators $D_{ \pm}^{(s)}$ coincides with those defined in (3.11) and the eigenfunctions $Y_{i}^{s, \pm}$ are nothing but the monopole harmonics $Y_{j m}^{s \pm 1 / 2}$. The monopole harmonics with $j=|s|-1 / 2$ are the fermion zeromodes. 
Summary. The one-loop determinant for general chiral multiplet of the R-charge $\mathrm{R}_{\mathrm{V}}=$ $2 q$ in the representation $\Lambda$ of the gauge group is,

$$
Z_{\mathrm{ch}, \Lambda}(s, a, q)=\prod_{w \in \Lambda} Z_{1-\operatorname{loop}}(s \cdot w, a \cdot w, q)=\prod_{w \in \Lambda} \frac{\Gamma(s \cdot w-i a \cdot w+q)}{\Gamma(s \cdot w+i a \cdot w+1-q)},
$$

where the saddle-point parameters $a, s$ are Cartan subalgebra valued, and $w$ runs over the weight vectors of the representation $\Lambda$. For the vector multiplets for simple Lie algebras the determinant is

$$
Z_{\mathrm{vec}}(s, a)=Z_{\mathrm{ch}, \text { adj }}(s, a, q=1)=\prod_{\alpha \in \Delta_{+}}\left\{(a \cdot \alpha)^{2}+(s \cdot \alpha)^{2}\right\},
$$

where the product runs over positive roots. The Coulomb branch localization formula for the partition function thus becomes

$$
Z_{S^{2}}=\frac{1}{|W|} \sum_{s} \int \frac{d^{r} a}{(2 \pi)^{r}} z^{\operatorname{Tr}(i a-s)} \bar{z}^{\operatorname{Tr}(i a+s)} Z_{\mathrm{vec}}(s, a) Z_{\mathrm{ch}, \Lambda}(s, a, q),
$$

where $z=\exp (-2 \pi \zeta-i \theta)$ and $r$ is the rank of the gauge group. For theories with flavor symmetries, one can introduce matter masses by gauging it by an external vector multiplet and turning on its $\sigma$-components. The matter one-loop determinant will then depend on the masses in an obvious manner.

Let us note here that, due to the anomaly in the antipodal map (3.27), the partition function is not invariant under a simple sign-flip of $\theta$ angle or $z \leftrightarrow \bar{z}$. For example, for a $\mathrm{U}(1)$ gauge theory with $N_{\mathrm{F}}$ electrons (chiral multiplets with charge +1 ) and $N_{\mathrm{A}}$ positrons (charge -1 ), the one-loop determinant from the matters obeys

$$
Z_{1 \text {-loop }}(s, a)=(-1)^{2 s\left(N_{\mathrm{F}}+N_{\mathrm{A}}\right)} Z_{1-\text { loop }}(-s, a),
$$

so the partition function is invariant under $z \rightarrow(-1)^{N_{\mathrm{F}}+N_{\mathrm{A}}} \bar{z}$.

\section{Defects}

In this section we wish to generalize the exact results for partition function to include the codimension two vortex defects. To keep the supersymmetry (2.4) unbroken, we only consider the defects inserted at the north and the south poles of the squashed sphere. The defects of interest are characterized by the singular behavior of the gauge field,

$$
A \simeq \eta^{\mathrm{N}} \cdot d \varphi(\theta=0), \quad A \simeq \eta^{\mathrm{S}} \cdot d \varphi \quad(\theta=\pi),
$$

where the constants $\eta^{\mathrm{N}}, \eta^{\mathrm{S}}$ are in Cartan subalgebra. In local Cartesian coordinates $x^{1}, x^{2}$ centered at the poles we have $F_{12} \simeq 2 \pi \eta^{\mathrm{N}} \delta^{2}(x)$ or $-2 \pi \eta^{\mathrm{S}} \delta^{2}(x)$. Noticing that our Killing spinors $\xi, \bar{\xi}$ are purely chiral or anti-chiral at the poles, the unbroken supersymmetry requires $D$ to take singular values,

$$
D \simeq 2 \pi i \eta^{\mathrm{N}} \delta^{2}(x) \quad(\theta=0), \quad D \simeq 2 \pi i \eta^{\mathrm{S}} \delta^{2}(x) \quad(\theta=\pi) .
$$


Abelian theories. Let us begin with the simplest case of the U(1) gauge theory. Up to gauge equivalence, the saddle point configurations in the presence of the vortex defects are given by

$$
\begin{aligned}
& \sigma=\frac{a}{\ell}, \quad D=\frac{a}{f \ell}+2 \pi i \eta^{\mathrm{N}} \delta_{(\mathrm{NP})}^{2}+2 \pi i \eta^{\mathrm{S}} \delta_{(\mathrm{SP})}^{2}, \\
& \rho=-\frac{s}{\ell}, \quad A= \begin{cases}s(\cos \theta-1) d \varphi+\eta^{\mathrm{N}} d \varphi, & \text { (north patch) } \\
s(\cos \theta+1) d \varphi+\eta^{\mathrm{S}} d \varphi . & \text { (south patch) }\end{cases}
\end{aligned}
$$

The quantization condition of magnetic flux gives $\eta^{\mathrm{N}}-\eta^{\mathrm{S}}-2 s \in \mathbb{Z}$. Using Coulomb branch localization, the correlators of the vortex defects are thus expressed as

$$
\left\langle V_{\eta^{\mathrm{N}}} V_{\eta^{\mathrm{S}}}\right\rangle=\sum_{s \in \frac{1}{2}\left(\eta^{\mathrm{N}}-\eta^{\mathrm{S}}+\mathbb{Z}\right)} \int d a z^{\eta^{\mathrm{N}}+i a-s} \bar{z}^{\eta^{\mathrm{S}}+i a+s} Z_{1 \text {-loop }},
$$

For the theories with $\mathrm{U}(1)$ gauge group, non-trivial one-loop determinant arises only from charged chiral multiplets. We would like to evaluate it by generalizing the analysis of the previous section.

The square of the supersymmetry on this saddle point is

$$
\mathbf{Q}^{2}=\frac{1}{\ell}\left\{\partial_{\varphi}+\frac{i}{2} \mathrm{R}_{\mathrm{V}}+\operatorname{Gauge}(\tilde{a})\right\}, \quad \tilde{a}= \begin{cases}a+i s-i \eta^{\mathrm{N}} & \text { (north patch) } \\ a-i s-i \eta^{\mathrm{S}} & \text { (south patch) }\end{cases}
$$

By a naive application of the fixed point formula, the contribution of a chiral multiplet with unit charge and $\mathrm{R}_{\mathrm{V}}=2 q$ to the determinant would be obtained from the character,

$$
\chi=\frac{x^{s-\eta^{\mathrm{N}}-i a+q}}{1-x}+\frac{x^{-s-\eta^{\mathrm{S}}-i a+q}}{1-x}=\sum_{n \geq 0}\left(x^{n+s-\eta^{\mathrm{N}}-i a+q}-x^{-n-s-\eta^{\mathrm{S}}-i a+q-1}\right) .
$$

In the second equality we expanded the first term in the left hand side into positive series in $x$ and the second term into the negative series. The other way of expansion gives the same answer thanks to the flux quantization condition. Assuming this is the correct character, one can translate it into the determinant

$$
Z_{1-\text { loop }}=\frac{\Gamma\left(s-\eta^{\mathrm{N}}-i a+q\right)}{\Gamma\left(s+\eta^{\mathrm{S}}+i a-q+1\right)} .
$$

This would mean that the effect of vortex defects in one-loop determinants is simply to shift the saddle-point parameters. Similar results were obtained for the expectation values of vortex loop observables in 3D SUSY gauge theories in [31, 32].

A problem with this naive result is that it does not respect the fact that $\eta^{\mathrm{N}}, \eta^{\mathrm{S}}$ are periodic parameters with period 1. Namely, under the assumption that the $\mathrm{U}(1)$ charges of all the matters are integers, one can shift $\eta^{\mathrm{N}}, \eta^{\mathrm{S}}$ by integers by singular gauge transformations without introducing multi-valuedness to the fields. Also, the normalizability of the modes (such as $Y_{i}^{s, \pm}$ in (3.30)) would not change under such gauge transformations, so the determinants should be invariant. The above naive formula should therefore be at most correct only for small $\eta^{\mathrm{N}}, \eta^{\mathrm{S}}$.

In the following we will find the fully general formula with the correct periodicity in $\eta$ 's, by making use of the correspondence between the gauge theory in the presence of codimension two defects (ramified bundle) and the gauge theory on orbifolds. 
Comparison with SUSY orbifolds. Let us consider the special case

$$
\eta^{\mathrm{N}}=\eta^{\mathrm{S}}=\eta=r / K(r, K \in \mathbb{Z}) .
$$

In this case, the singularity of the gauge field at the two poles can be removed by the gauge transformation $A^{\prime}=A-\eta d \varphi$. But this makes the charged matter fields non-periodic around the poles. For example, the matter field $\Phi(\theta, \varphi)$ with unit $\mathrm{U}(1)$ charge obeys twisted periodicity condition,

$$
\Phi(\theta, \varphi+2 \pi)=\Phi(\theta, \varphi) e^{-2 \pi i \eta} .
$$

Recall that the terms in the character (3.24) obtained in the previous section were associated with the basis of local holomorphic functions at the two poles. The $\eta$-dependence of the character (4.6) on the defect background can be understood to arise because the basis holomorphic functions now have to obey twisted periodicity condition.

We recall that similar twist in periodicity appears in gauge theories on the orbifold $S^{2} / \mathbb{Z}_{K}$, where the $\mathbb{Z}_{K}$ symmetry act on charged fields as a gauge rotation as well as the rotation of the sphere

$$
\Phi\left(\theta, \varphi+\frac{2 \pi}{K}\right)=\Phi(\theta, \varphi) e^{-\frac{2 \pi i r}{K}} .
$$

The orbifolds of sphere can be regarded as squashed spheres with conical singularities at the two poles. Our idea is thus to compute the correlators of vortex defects (4.4) as an orbifold partition function, by assuming the SUSY partition function to be independent of squashings even with conical singularities.

We are interested in the orbifold of $S^{2}$ preserving supersymmetry. Our Killing spinors $\xi, \bar{\xi}(2.4)$ are not invariant under the $\mathbb{Z}_{K}$ rotation $\varphi \rightarrow \varphi+\frac{2 \pi}{K}$, but they satisfy

$$
\xi\left(\theta, \varphi+\frac{2 \pi}{K}\right)=e^{-\frac{i \pi}{K}} \xi(\theta, \varphi), \quad \bar{\xi}\left(\theta, \varphi+\frac{2 \pi}{K}\right)=e^{\frac{i \pi}{K} \bar{\xi}(\theta, \varphi)}
$$

So the supersymmetric $\mathbb{Z}_{K}$ orbifold should involve the vector R-symmetry rotation. At first sight it seems natural to impose the orbifold projection

$$
\Phi\left(\theta, \varphi+\frac{2 \pi}{K}\right) \stackrel{?}{=} e^{-\frac{i \pi}{K} \cdot \mathrm{R}_{\mathrm{V}}-\frac{2 \pi i r}{K} \cdot Q} \Phi(\theta, \varphi)
$$

on the field $\Phi$ of electric charge $Q$. However, this condition is not consistent with the periodicity of the fields on the sphere before orbifolding,

$$
\Phi(\theta, \varphi+2 \pi)=(-1)^{2 \times(\operatorname{spin})} \Phi(\theta, \varphi),
$$

where the sign factor arises because of our choice of local Lorentz frame in which the spin connection takes the form (2.5). It turns out that the SUSY-preserving orbifold which is suitable for our purpose is

$$
\Phi\left(\theta, \varphi+\frac{2 \pi}{K}\right)=(-1)^{2 \times(\operatorname{spin})} e^{\frac{i \pi(K-1)}{K} \cdot \mathrm{R}_{\mathrm{V}}-\frac{2 \pi i r}{K} \cdot Q} \Phi(\theta, \varphi) .
$$


In order for this to be a $\mathbb{Z}_{K}$ orbifold projection, one also needs to impose that $K$ is odd and the label $q$ of all the chiral multiplets has to satisfy $(K-1) q \in \mathbb{Z}$. But even if these conditions are not met, the condition (4.14) can be interpreted as the periodicity condition for the fields on a squashed sphere,

$$
d s^{2}=f^{2} d \theta^{2}+\ell^{2} \sin ^{2} \theta d \varphi^{2}, \quad V=\frac{1}{2}\left(\frac{\ell}{f}-1\right) d \varphi, \quad(f \equiv K \ell)
$$

with vortex defects at the two poles. The $\mathrm{R}_{\mathrm{V}}$-charge dependence of the projection condition (4.14) arises after one gauges away the background field $V$. This interpretation works for arbitrary real positive $K$.

Let us work out the orbifolded character for a chiral multiplet with unit electric charge and $\mathrm{R}_{\mathrm{V}}=2 q$. The projection condition on the variables $\mathbf{X}, \boldsymbol{\Xi}$ becomes

$$
\begin{aligned}
& \mathbf{X}\left(\theta, \varphi+\frac{2 \pi}{K}\right)=e^{\frac{2 \pi i}{K}(K-1) q-\frac{2 \pi i}{K} r} \mathbf{X}(\theta, \varphi), \\
& \boldsymbol{\Xi}\left(\theta, \varphi+\frac{2 \pi}{K}\right)=e^{\frac{2 \pi i}{K}(K-1)(q-1)-\frac{2 \pi i}{K} r} \boldsymbol{\Xi}(\theta, \varphi) .
\end{aligned}
$$

We assume $(K-1) q$ is an integer, and also that the R-charge takes a reasonable value $0 \leq 2 q \leq 2$. The orbifolded character is then obtained by projecting to the $\mathbb{Z}_{K}$-invariant modes,

$$
\chi=\sum_{n \geq 0}^{\infty} x^{n+s-i a+q}-\sum_{n \geq 0}^{\odot} x^{-n-s-i a+q-1} \cdot\left(\begin{array}{l}
\boldsymbol{\omega}: n=q(K-1)-r \bmod K \\
\odot: n=-(q-1)(K-1)+r \bmod K
\end{array}\right)
$$

By introducing the rescaled variables

$$
x^{K}=\tilde{x}, \quad \frac{r}{K}=\eta, \quad \frac{s}{K}=\tilde{s}, \quad \frac{a}{K}=\tilde{a},
$$

one can rewrite the character as follows,

$$
\chi=\sum_{n \geq \eta-q+\frac{q}{K}, n \in \mathbb{Z}} \tilde{x}^{n+\tilde{s}-\eta-i \tilde{a}+q}-\sum_{n \leq \eta-q-\frac{1-q}{K}, n \in \mathbb{Z}} \tilde{x}^{n-\tilde{s}-\eta-i \tilde{a}+q} .
$$

The rescaling of $x$ is to adjust the radius of the orbifold $S^{2} / \mathbb{Z}_{K}$ to that of the squashed sphere (4.15). Note also that $s$ is the magnetic flux through the $S^{2}$, whereas the flux through the orbifold is $\tilde{s}$ which is to be GNO quantized.

In terms of the rescaled variables, the orbifolded character is almost independent of $K$. The only $K$-dependence remains in the condition of the summation over $n$, and it can be dropped in the limit of large $K$ (which is required so that $\eta$ can be well approximated by a rational number $r / K$ ) as long as $\eta-q$ is not an integer. Based on the above result we propose the formula for the character on the defect background,

$$
\begin{aligned}
& \chi=\sum_{n \geq \eta-q, n \in \mathbb{Z}} x^{n+s-\eta-i a+q}-\sum_{n<\eta-q, n \in \mathbb{Z}} x^{n-s-\eta-i a+q}\left(q<\frac{1}{2}\right), \\
& \chi=\sum_{n>\eta-q, n \in \mathbb{Z}} x^{n+s-\eta-i a+q}-\sum_{n \leq \eta-q, n \in \mathbb{Z}} x^{n-s-\eta-i a+q}\left(q>\frac{1}{2}\right) .
\end{aligned}
$$



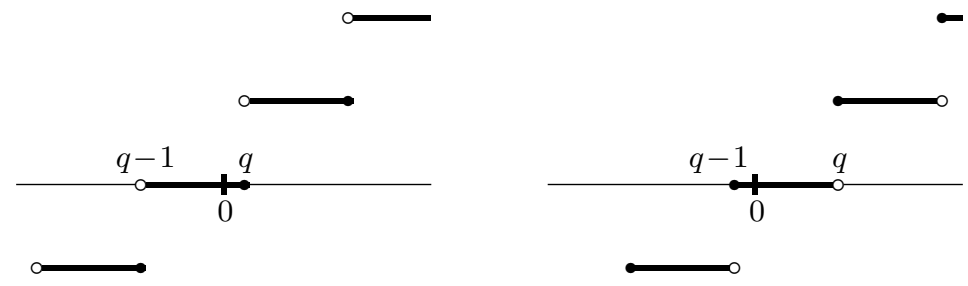

Figure 1. The function $[x]_{q}$ for $q<1 / 2$ (left) and $q>1 / 2$ (right).

Note that there is a subtlety when $\eta-q$ happens to be an integer, and we decided to treat those special cases so that the character just flips the sign under charge conjugation combined with $q \rightarrow 1-q$. Note also that the character has the expected periodicity in $\eta$. For more general defect backgrounds with $\eta^{\mathrm{N}} \neq \eta^{\mathrm{S}}$, we propose that the character and the corresponding one-loop determinant are periodic functions of $\eta^{\mathrm{N}}, \eta^{\mathrm{S}}$ with period 1 . They are given by

$$
\begin{aligned}
\chi & =\sum_{n \geq 0}\left(x^{n+s-\eta^{\mathrm{N}}-i a+q+\left[\eta^{\mathrm{N}}\right]_{q}}-x^{-n-s-\eta^{\mathrm{S}}-i a+q-1+\left[\eta^{\mathrm{S}}\right]_{q}}\right), \\
Z_{1-\text { loop }} & =(-1)^{\left[\eta^{\mathrm{S}}\right]_{q}} \frac{\Gamma\left(s-\eta^{\mathrm{N}}-i a+q+\left[\eta^{\mathrm{N}}\right]_{q}\right)}{\Gamma\left(1+s+\eta^{\mathrm{S}}+i a-q-\left[\eta^{\mathrm{S}}\right]_{q}\right)},
\end{aligned}
$$

where $[\eta]_{q}$ is an integer-valued function (see figure 1)

$$
[\eta]_{q} \equiv \begin{cases}\lceil\eta-q\rceil & \left(q<\frac{1}{2}\right) \\ \lfloor\eta-q+1\rfloor & \left(q>\frac{1}{2}\right)\end{cases}
$$

We chose to put a subtle sign factor to $Z_{1 \text {-loop }}$ so that the antipodal map, which sign-flips $s$ and exchanges $\eta^{\mathrm{N}}$ and $\eta^{\mathrm{S}}$, is an anomalous symmetry of the theory even in the presence of the vortex defects. Namely, (4.20) can be rewritten as

$$
Z_{1-\text { loop }}=(-1)^{2 s+\eta^{\mathrm{S}}-\eta^{\mathrm{N}}} \cdot(-1)^{\left[\eta^{\mathrm{N}}\right]_{q}} \frac{\Gamma\left(-s-\eta^{\mathrm{S}}-i a+q+\left[\eta^{\mathrm{S}}\right]_{q}\right)}{\Gamma\left(1-s+\eta^{\mathrm{N}}+i a-q-\left[\eta^{\mathrm{N}}\right]_{q}\right)}
$$

where the sign factor on the right hand side is understood to arise from the non-invariance of the measure. This sign factor can be expressed in terms of the magnetic flux, so it can be absorbed into a shift of the theta angle.

Example 1: SQED. As an application, let us study the vortex defects in the SQED with $N_{\mathrm{F}}$ electrons and $N_{\mathrm{A}}$ positrons. To make the formulae short, we combine the masses $\mu$ and the $\mathrm{R}_{\mathrm{V}}$-charges $q$ of the matters into complex parameters $m$,

$$
m_{i} \equiv \mu_{i}+i q_{i} \quad\left(i=1, \cdots, N_{\mathrm{F}}\right), \quad \tilde{m}_{i} \equiv \tilde{\mu}_{i}+i \tilde{q}_{i} \quad\left(i=1, \cdots, N_{\mathrm{A}}\right) .
$$


The correlator of the vortex defects can be expressed as

$$
\begin{aligned}
\left\langle V_{\eta^{\mathrm{N}}} V_{\eta^{\mathrm{S}}}\right\rangle= & \sum_{s \in \frac{1}{2}\left(\eta^{\mathrm{N}}-\eta^{\mathrm{S}}+\mathbb{Z}\right)} \int \frac{d a}{2 \pi} z^{-s+\eta^{\mathrm{N}}+i a} \bar{z}^{s+\eta^{\mathrm{S}}+i a} \cdot Z_{\mathrm{ch}}^{\mathrm{SQED}}, \\
Z_{\mathrm{ch}}^{\mathrm{SQED}} \equiv & \prod_{i=1}^{N_{\mathrm{F}}}(-1)^{\left[\eta^{\mathrm{S}}\right]_{q_{i}}} \frac{\Gamma\left(s-\eta^{\mathrm{N}}-i a-i m_{i}+\left[\eta^{\mathrm{N}}\right]_{q_{i}}\right)}{\Gamma\left(1+s+\eta^{\mathrm{S}}+i a+i m_{i}-\left[\eta^{\mathrm{S}}\right]_{q_{i}}\right)} \\
& \cdot \prod_{i=1}^{N_{\mathrm{A}}}(-1)^{\left[-\eta^{\mathrm{S}}\right]_{\tilde{q}_{i}}} \frac{\Gamma\left(-s+\eta^{\mathrm{N}}+i a-i \tilde{m}_{i}+\left[-\eta^{\mathrm{N}}\right]_{\tilde{q}_{i}}\right)}{\Gamma\left(1-s-\eta^{\mathrm{S}}-i a+i \tilde{m}_{i}-\left[-\eta^{\mathrm{S}}\right]_{\tilde{q}_{i}}\right)} .
\end{aligned}
$$

There are two questions we would like to address. One is whether the apparent dependence on the $\eta$ 's can be eliminated by a redefinition of contours, or there is nontrivial $\eta$-dependence remaining. The other is whether the Higgs branch expression has a factorized form as in the absence of the defects.

The contour of $a$-integration can be closed in the lower or upper half-planes depending on whether $|z|<1$ or $|z|>1[34,35]$. Let us focus on the former case. The contour integral then picks up the poles of the determinants of $N_{\mathrm{F}}$ electrons. The determinant of the $j$-th electron has a set of poles labeled by a pair of non-negative integers $\left(k_{\mathrm{N}}, k_{\mathrm{S}}\right)$. The saddle point parameters $a$ and $s$ are related to them by

$$
\begin{aligned}
-s+\eta^{\mathrm{N}}+i a & =-i m_{j}+\left[\eta^{\mathrm{N}}\right]_{q_{j}}+k_{\mathrm{N}}, \\
s+\eta^{\mathrm{S}}+i a & =-i m_{j}+\left[\eta^{\mathrm{S}}\right]_{q_{j}}+k_{\mathrm{S}} .
\end{aligned}
$$

One can check the value of $a$ is always in the lower half plane. The sum over residues can then be organized into the following form

$$
\begin{aligned}
\left\langle V_{\eta^{\mathrm{N}}} V_{\eta^{\mathrm{S}}}\right\rangle= & \sum_{j=1}^{N_{\mathrm{F}}} z^{-i m_{j}} \bar{z}^{-i m_{j}} \prod_{i \neq j}^{N_{\mathrm{F}}} \frac{\Gamma\left(-i m_{i}+i m_{j}\right)}{\Gamma\left(1+i m_{i}-i m_{j}\right)} \prod_{i=1}^{N_{\mathrm{A}}} \frac{\Gamma\left(-i \tilde{m}_{i}-i m_{j}\right)}{\Gamma\left(1+i \tilde{m}_{i}+i m_{j}\right)} \\
& \times F_{\text {vortex }}^{[j]}\left((-1)^{N_{\mathrm{F}}} z ; \eta^{\mathrm{N}}, m_{i}, \tilde{m}_{i}\right) F_{\text {vortex }}^{[j]}\left((-1)^{\left.N_{\mathrm{A}} \bar{z} ; \eta^{\mathrm{S}}, m_{i}, \tilde{m}_{i}\right)},\right.
\end{aligned}
$$

where $F_{\text {vortex }}^{[j]}$ is a generalization of the vortex partition function for the SQED,

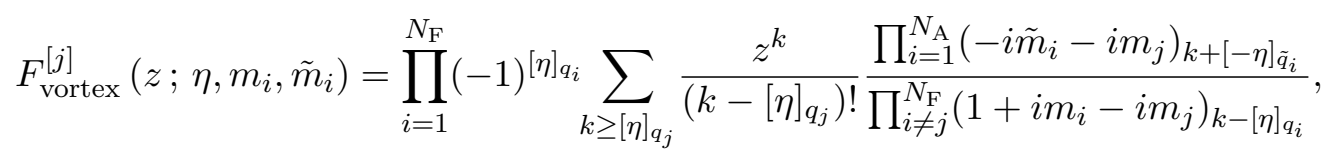

and $(x)_{n}=\Gamma(x+n) / \Gamma(x)$ is the Pochhammer symbol (we allow the integer $n$ to become negative). Note that, in the product of two vortex partition functions, one depends only on $\eta^{\mathrm{N}}$ while the other depends only on $\eta^{\mathrm{S}}$. Therefore, as expected, each vortex partition function captures the physics near one of the poles.

The correlator of the vortex defects thus depends on $\eta^{\mathrm{N}, \mathrm{S}}$ only through the integers $\left[\eta^{\mathrm{N}}\right]_{q_{i}}$, etc. The correlator jumps when $\eta^{\mathrm{N}}$ or $\eta^{\mathrm{S}}$ coincide with one of $q_{i}$ or $-\tilde{q}_{i}$ modulo integer. Note also that $F_{\text {vortex }}^{[j]}$ is periodic in $\eta$ up to multiplication by constants and powers of $z$. 
Non-Abelian theories. Let us next turn to non-Abelian gauge theories. The parameters of the vortex defects $\boldsymbol{\eta}^{\mathrm{N}}, \boldsymbol{\eta}^{\mathrm{S}}$ and the saddle point parameters $\boldsymbol{s}, \boldsymbol{a}$ now take values in Cartan subalgebra. By generalizing the result for the abelian case, we find the character for a chiral multiplet in the representation $\Lambda$ and $\mathrm{R}_{\mathrm{V}}=q$ is given by

$$
\chi_{\Lambda, q}=\sum_{w \in \Lambda} \sum_{n \geq 0}\left(x^{n+q+w \cdot\left(\boldsymbol{s}-\boldsymbol{\eta}^{\mathrm{N}}-i \boldsymbol{a}\right)+\left[w \cdot \boldsymbol{\eta}^{\mathrm{N}}\right]_{q}}-x^{-n+q-1-w \cdot\left(\boldsymbol{s}+\boldsymbol{\eta}^{\mathrm{S}}+i \boldsymbol{a}\right)+\left[w \cdot \boldsymbol{\eta}^{\mathrm{S}}\right]_{q}}\right),
$$

and the determinant is

$$
Z_{\mathrm{ch}, \Lambda}=\prod_{w \in \Lambda}(-1)^{\left[w \cdot \eta^{\mathrm{S}}\right]_{q}} \frac{\Gamma\left(q+w \cdot\left(\boldsymbol{s}-\boldsymbol{\eta}^{\mathrm{N}}-i \boldsymbol{a}\right)+\left[w \cdot \boldsymbol{\eta}^{\mathrm{N}}\right]_{q}\right)}{\Gamma\left(1-q+w \cdot\left(\boldsymbol{s}+\boldsymbol{\eta}^{\mathrm{S}}+i \boldsymbol{a}\right)-\left[w \cdot \boldsymbol{\eta}^{\mathrm{S}}\right]_{q}\right)} .
$$

The formula for the vector multiplet is obtained by setting $\Lambda=\operatorname{adj}$ and $q=1$.

For concreteness, let us consider the $\mathrm{U}(N)$ gauge group in the following. The parameters $\boldsymbol{\eta}^{\mathrm{N}}, \boldsymbol{\eta}^{\mathrm{S}}, \boldsymbol{s}$ and $\boldsymbol{a}$ are now $N \times N$ diagonal matrices,

$$
\begin{aligned}
\boldsymbol{\eta}^{\mathrm{N}}=\operatorname{diag}\left(\eta_{1}^{\mathrm{N}}, \cdots, \eta_{N}^{\mathrm{N}}\right), & \boldsymbol{s}=\operatorname{diag}\left(s_{1}, \cdots, s_{N}\right), \\
\boldsymbol{\eta}^{\mathrm{S}}=\operatorname{diag}\left(\eta_{1}^{\mathrm{S}}, \cdots, \eta_{N}^{\mathrm{S}}\right), & \boldsymbol{a}=\operatorname{diag}\left(a_{1}, \cdots, a_{N}\right) .
\end{aligned}
$$

As in the abelian case, $s$ is subject to the flux quantization condition $\boldsymbol{\eta}^{\mathrm{N}}-\boldsymbol{\eta}^{\mathrm{S}}-2 \boldsymbol{s} \in \mathbb{Z}^{N}$. The defects at the poles break the gauge symmetry to a subgroup called Levi subgroup. Generically $\mathrm{U}(N)$ is broken to $\mathrm{U}(1)^{N}$, but some non-Abelian symmetry remains if the diagonal elements of $\boldsymbol{\eta}^{\mathrm{N}}$ or $\boldsymbol{\eta}^{\mathrm{S}}$ degenerate.

The Coulomb branch localization in the presence of non-Abelian vortex defects has an important subtlety: the localized path integration involves summation over Weyl images of $\boldsymbol{\eta}^{\mathrm{N}}$ and $\boldsymbol{\eta}^{\mathrm{S}}$, though the two saddle points related to each other by a simultaneous Weyl reflections of $\boldsymbol{a}, \boldsymbol{s}, \boldsymbol{\eta}^{\mathrm{N}}, \boldsymbol{\eta}^{\mathrm{S}}$ are of course equivalent.

The character for the $\mathrm{U}(N)$ vector multiplet is given by

$$
\begin{aligned}
\chi= & \sum_{\eta_{a b}^{\mathrm{N}} \geq 0} \sum_{n \geq 0} x^{n+s_{a b}-\eta_{a b}^{\mathrm{N}}-i a_{a b}+1}-\sum_{\eta_{a b}^{\mathrm{S}} \geq 0} \sum_{n \geq 0} x^{-n-s_{a b}-\eta_{a b}^{\mathrm{S}}-i a_{a b}} \\
& +\sum_{\eta_{a b}^{\mathrm{N}}<0} \sum_{n \geq 0} x^{n+s_{a b}-\eta_{a b}^{\mathrm{N}}-i a_{a b}}-\sum_{\eta_{a b}^{\mathrm{S}}<0} \sum_{n \geq 0} x^{-n-s_{a b}-\eta_{a b}^{\mathrm{S}}-i a_{a b}-1},
\end{aligned}
$$

where we used the short-hand notation $s_{a b} \equiv s_{a}-s_{b}$, etc., and assumed $\left|\eta_{a b}^{\mathrm{N}}\right|,\left|\eta_{a b}^{\mathrm{S}}\right|<1$ for simplicity. It turns out that the corresponding determinant takes the following simple form,

$$
\begin{aligned}
Z_{\mathrm{vec}}= & (-1)^{\frac{1}{2} N(N-1)}(-1)^{(N-1) \operatorname{Tr}\left(2 \boldsymbol{s}+\boldsymbol{\eta}^{\mathrm{S}}-\boldsymbol{\eta}^{\mathrm{N}}\right)} \\
& \times \sigma\left(\boldsymbol{\eta}^{\mathrm{N}}\right) \sigma\left(\boldsymbol{\eta}^{\mathrm{S}}\right) \prod_{a<b, \eta_{a b}^{\mathrm{N}}=0}\left(s_{a b}-i a_{a b}\right) \cdot \prod_{a<b, \eta_{a b}^{\mathrm{S}}=0}\left(-s_{a b}-i a_{a b}\right) .
\end{aligned}
$$

Here $\sigma\left(\boldsymbol{\eta}^{\mathrm{N}}\right)$ is the parity of the unique permutation $\pi$ which brings $\left(\eta_{1}^{\mathrm{N}}, \cdots, \eta_{N}^{\mathrm{N}}\right)$ to the standard order satisfying

$$
\pi(a)<\pi(b) \Longrightarrow\left(\eta_{\pi(a)}^{\mathrm{N}}<\eta_{\pi(b)}^{\mathrm{N}} \quad \text { or } \quad\left(\eta_{\pi(a)}^{\mathrm{N}}=\eta_{\pi(b)}^{\mathrm{N}}, \quad a<b\right)\right)
$$


and similarly for $\sigma\left(\boldsymbol{\eta}^{\mathrm{S}}\right)$. The second sign factor in the right hand side of (4.31) can be absorbed into the redefinition of the FI-theta parameter, $z(-1)^{N-1} \rightarrow z$.

The discontinuity of the determinant is at $\eta_{a b}^{\mathrm{N}}=0$ or $\eta_{a b}^{\mathrm{S}}=0$, namely when the eivenvalues of $\boldsymbol{\eta}^{\mathrm{N}, \mathrm{S}}$ degenerate. The properties of vortex defects are therefore qualitatively different depending on the choice of Levi subgroups. Let us now take two vortex defects $V_{\boldsymbol{\eta}^{\mathrm{N}}}, V_{\boldsymbol{\eta}^{\mathrm{S}}}$ corresponding to the Levi subgroups $\mathbb{L}^{\mathrm{N}}$ and $\mathbb{L}^{\mathrm{S}}$, and take the parameters $\boldsymbol{\eta}^{\mathrm{N}}, \boldsymbol{\eta}^{\mathrm{S}}$ to be vanishingly small. In this limit, the one-loop determinant of vector multiplet does not agree with the one in the absence of the defect. It rather satisfies

$$
Z_{\mathrm{vec}}\left(\mathbb{L}^{\mathrm{N}}, \mathbb{L}^{\mathrm{S}} ; \boldsymbol{\eta}^{\mathrm{N}}, \boldsymbol{\eta}^{\mathrm{S}}\right) \stackrel{\boldsymbol{\eta}^{\mathrm{N}}, \boldsymbol{\eta}^{\mathrm{S}} \rightarrow 0}{\longrightarrow} Z_{\mathrm{vec}}\left(\boldsymbol{\eta}^{\mathrm{N}}=\boldsymbol{\eta}^{\mathrm{S}}=0\right) \cdot \prod_{\substack{\alpha \in \Delta_{+} \\ \alpha \notin \mathbb{L}^{\mathrm{N}}}} \frac{1}{\alpha \cdot(s-i a)} \cdot \prod_{\substack{\alpha \in \Delta_{+} \\ \alpha \notin \mathbb{L}^{\mathrm{S}}}} \frac{1}{\alpha \cdot(s+i a)}
$$

The products are over the positive roots of $\mathrm{U}(N)$ which are broken due to the defects. It is interesting to recall here that $s \mp i a$ are proportional to the saddle point values of $\sigma \mp i \rho$. The relation (4.33) therefore implies that the vortex defects have the same effect as inserting certain local operators made of $\sigma-i \rho$ at the north pole, $\sigma+i \rho$ at the south pole. Usually we require such operators to be gauge invariant, such as

$$
\operatorname{Tr}(\sigma \mp i \rho)^{j}
$$

The local operators made of $\sigma \mp i \rho$ here, on the other hand, are not gauge invariant, but they are allowed because the gauge symmetry is broken at the poles because of the defects.

An important special case is

$$
\boldsymbol{\eta}^{\mathrm{N}}=\boldsymbol{\eta}^{\mathrm{S}}=(\underbrace{\frac{1}{K}, \ldots, \frac{1}{K}}_{n_{1} \text { times }}, \underbrace{\frac{2}{K}, \ldots, \frac{2}{K}}_{n_{2} \text { times }}, \cdots),
$$

which can be understood also as an orbifold $S^{2} / \mathbb{Z}_{K}$ breaking $\mathrm{U}(N)$ to $\mathbb{L} \equiv \prod \mathrm{U}\left(n_{i}\right)$. The one-loop determinant (4.31) for this case is nothing but the determinant for the gauge group $\mathbb{L}$ in the absence of defects.

Example 2: SQCD. As an application of our formalism, let us work out the defect correlators for the $\mathrm{U}(N)$ SQCD with $N_{\mathrm{F}}$ fundamental and $N_{\mathrm{A}}$ anti-fundamental matters. As in the case of SQED, we denote the masses and $\mathrm{R}_{\mathrm{V}}$-charges of the $N_{\mathrm{F}}$ fundamentals as $m_{j}=\mu_{j}+i q_{j}$, and those of the $N_{\mathrm{A}}$ anti-fundamentals as $\tilde{m}_{j}=\tilde{\mu}_{j}+i \tilde{q}_{j}$.

We begin by summarizing the result for SQED to introduce some notations. For $|z|<1$ the contour of $a$-integral can be closed in the lower half plane, and we obtained the formula

$$
\begin{aligned}
\left\langle V_{\eta^{\mathrm{N}}} V_{\eta^{\mathrm{S}}}\right\rangle^{\mathrm{SQED}}(z, \bar{z}) & =\sum_{s} \int \frac{d a}{2 \pi} z^{\eta^{\mathrm{N}}+i a-s} \bar{z}^{\eta^{\mathrm{S}}+i a+s} \cdot Z_{\mathrm{ch}}^{\mathrm{SQED}}\left(s, a, \eta^{\mathrm{N}}, \eta^{\mathrm{S}}\right) \\
& =\sum_{j=1}^{N_{\mathrm{F}}} Z_{j} \cdot F_{j}\left(z, \eta^{\mathrm{N}}\right) \cdot \bar{F}_{j}\left(\bar{z}, \eta^{\mathrm{S}}\right),
\end{aligned}
$$


where

$$
\begin{aligned}
Z_{j} & \equiv \prod_{i \neq j}^{N_{\mathrm{F}}} \frac{\Gamma\left(-i m_{i}+i m_{j}\right)}{\Gamma\left(1+i m_{i}-i m_{j}\right)} \prod_{i=1}^{N_{\mathrm{A}}} \frac{\Gamma\left(-i \tilde{m}_{i}-i m_{j}\right)}{\Gamma\left(1+i \tilde{m}_{i}+i m_{j}\right)}, \\
F_{j}(z, \eta) & \equiv z^{-i m_{j}} F_{\text {vortex }}^{[j]}\left((-1)^{N_{\mathrm{F}}} z ; \eta, m_{i}, \tilde{m}_{i}\right), \\
\bar{F}_{j}(\bar{z}, \eta) & \equiv \bar{z}^{-i m_{j}} F_{\text {vortex }}^{[j]}\left((-1)^{N_{\mathrm{A}}} \bar{z} ; \eta, m_{i}, \tilde{m}_{i}\right),
\end{aligned}
$$

and $F_{\text {vortex }}^{j}$ has been defined in (4.27). In the second line of (4.35), the dependence on the mass parameters $m_{i}, \tilde{m}_{i}$ or the $\mathrm{R}_{\mathrm{V}}$-charges $q_{i}, \tilde{q}_{i}$ have been suppressed for later convenience.

To write down the defect correlators in the $\mathrm{U}(N)$ SQCD, we first recall the trick to rewrite the one-loop determinant of the vector multiplet by differentiations.

$$
\begin{aligned}
& z^{\operatorname{Tr}\left(\boldsymbol{\eta}^{\mathrm{N}}+i \boldsymbol{a}-\boldsymbol{s}\right)} \bar{z}^{\operatorname{Tr}\left(\boldsymbol{\eta}^{\mathrm{S}}+i \boldsymbol{a}+\boldsymbol{s}\right)} \cdot \prod_{a<b, \eta_{a b}^{\mathrm{N}}=0}\left(s_{a b}-i a_{a b}\right) \prod_{a<b, \eta_{a b}^{\mathrm{S}}=0}\left(-s_{a b}-i a_{a b}\right) \\
& \quad=\prod_{a<b, \eta_{a b}^{\mathrm{N}}=0}\left(z_{b} \partial_{z_{b}}-z_{a} \partial_{z_{a}}\right) \prod_{a<b, \eta_{a b}^{\mathrm{S}}=0}\left(\bar{z}_{b} \partial_{\bar{z}_{b}}-\bar{z}_{a} \partial_{\bar{z}_{a}}\right)\left[\prod_{a=1}^{N} z_{a}^{\left(\eta^{\mathrm{N}}+i a-s\right)_{a}} \bar{z}_{a}^{\left(\eta^{\mathrm{S}}+i a+s\right)_{a}}\right] \\
& \bar{z}_{a} \rightarrow \bar{z} \\
&
\end{aligned} .
$$

The defect correlator in the SQCD can therefore be obtained by first acting the above differential operator onto the correlator of $\mathrm{U}(1)^{N}$ theory,

$$
\prod_{a<b, \eta_{a b}^{\mathrm{N}}=0}\left(z_{b} \partial_{z_{b}}-z_{a} \partial_{z_{a}}\right) \cdot \prod_{a<b, \eta_{a b}^{\mathrm{S}}=0}\left(\bar{z}_{b} \partial_{\bar{z}_{b}}-\bar{z}_{a} \partial_{\bar{z}_{a}}\right) \prod_{a=1}^{N}\left\langle V_{\eta_{a}^{\mathrm{N}}} V_{\eta_{a}^{\mathrm{S}}}\right\rangle^{\mathrm{SQED}}\left(z_{a}, \bar{z}_{a}\right),
$$

and then setting the $N$ FI-theta parameters to be all equal,

$$
z_{a} \rightarrow(-1)^{N-1} z \equiv \hat{z} .
$$

We also need to sum over the Weyl images of $\boldsymbol{\eta}^{\mathrm{N}}, \boldsymbol{\eta}^{\mathrm{S}}$ with the weight $\sigma\left(\boldsymbol{\eta}^{\mathrm{N}}\right) \sigma\left(\boldsymbol{\eta}^{\mathrm{S}}\right)$. In the following we write down the formulae for defect correlators explicitly for two special cases. The generalization is straightforward.

Case 1. One of the simplest special cases is when $\boldsymbol{\eta}^{\mathrm{N}}, \boldsymbol{\eta}^{\mathrm{S}}$ are both nonzero but proportional to the identity, namely $\eta_{a}^{\mathrm{N}}=\eta^{\mathrm{N}}$ and $\eta_{a}^{\mathrm{S}}=\eta^{\mathrm{S}}$ for all $a$. Since $\boldsymbol{\eta}^{\mathrm{N}}$ and $\boldsymbol{\eta}^{\mathrm{S}}$ are Weyl-reflection invariant, we only need to take the differentiation of the product of SQED correlators.

$$
\begin{aligned}
\left\langle V_{\boldsymbol{\eta}^{\mathrm{N}}} V_{\eta^{\mathrm{S}}}\right\rangle^{\mathrm{SQCD}} & =\left.\frac{1}{N !} \prod_{a<b}^{N}\left|z_{b} \partial_{z_{b}}-z_{a} \partial_{z_{a}}\right|^{2} \sum_{j_{a}=1}^{N_{\mathrm{F}}} \prod_{a=1}^{N} Z_{j_{a}} F_{j_{a}}\left(z_{a}, \eta^{\mathrm{N}}\right) \bar{F}_{j_{a}}\left(\bar{z}_{a}, \eta^{\mathrm{S}}\right)\right|_{z_{a} \rightarrow \hat{z}} \\
& =\sum_{\left\{j_{a}\right\}} \prod_{a=1}^{N} Z_{j_{a}} \cdot \operatorname{det}\left[F_{j_{a}}^{[b]}\left(\hat{z}, \eta^{\mathrm{N}}\right)\right] \cdot \operatorname{det}\left[F_{j_{a}}^{[b]}\left(\overline{\hat{z}}, \eta^{\mathrm{S}}\right)\right] .
\end{aligned}
$$

Here the determinant is that of $N \times N$ matrices with the $(a, b)$-th entry

$$
F_{j_{a}}^{[b]}(z, \eta) \equiv\left(z \partial_{z}\right)^{b-1} F_{j_{a}}(z, \eta) .
$$

The summation in (4.38) is thus over the sets of $N$ different integers chosen from $\left\{1, \cdots, N_{\mathrm{F}}\right\}$. 
Case 2. Another special case is when $\boldsymbol{\eta}^{\mathrm{N}}, \boldsymbol{\eta}^{\mathrm{S}}$ are both generic diagonal matrices with no degeneration of eigenvalues. Then the one-loop determinant of the vector multiplet is essentially $\sigma\left(\boldsymbol{\eta}^{\mathrm{N}}\right) \sigma\left(\boldsymbol{\eta}^{\mathrm{S}}\right)$. There is no need of differentiation, but we need to sum over all the Weyl images of $\boldsymbol{\eta}^{\mathrm{N}}$ and $\boldsymbol{\eta}^{\mathrm{S}}$. We thus obtain

$$
\begin{aligned}
\left\langle V_{\boldsymbol{\eta}^{\mathrm{N}}} V_{\boldsymbol{\eta}^{\mathrm{S}}}\right\rangle^{\mathrm{SQCD}} & =\left.\frac{1}{N !} \sum_{\pi, \bar{\pi} \in S_{N}} \operatorname{sgn}(\pi) \cdot \operatorname{sgn}(\bar{\pi}) \cdot \sum_{j_{a}=1}^{N_{\mathrm{F}}} \prod_{a=1}^{N} Z_{j_{a}} F_{j_{a}}\left(z_{a}, \eta_{\pi(a)}^{\mathrm{N}}\right) \bar{F}_{j_{a}}\left(\bar{z}_{a}, \eta_{\bar{\pi}(a)}^{\mathrm{S}}\right)\right|_{z_{a} \rightarrow \hat{z}} \\
& =\sum_{\left\{j_{a}\right\}} \prod_{a=1}^{N} Z_{j_{a}} \cdot \operatorname{det}\left[F_{j_{a}}\left(\hat{z}, \eta_{b}^{\mathrm{N}}\right)\right] \cdot \operatorname{det}\left[F_{j_{a}}\left(\overline{\hat{z}}, \eta_{b}^{\mathrm{S}}\right)\right] .
\end{aligned}
$$

Again, the summation is over the set of $N$ different integers $\left\{j_{a}\right\}$. Note also that the matrix elements $F_{j_{a}}\left(z, \eta_{b}\right)$, as a functions of $\eta_{b}$, are locally constant. In particular, if none of $q_{i}$ or $-\tilde{q}_{i}$ is in between $\eta_{b}^{\mathrm{N}}$ and $\eta_{c}^{\mathrm{N}}$, then

$$
F_{j_{a}}\left(\hat{z}, \eta_{b}^{\mathrm{N}}\right)=F_{j_{a}}\left(\hat{z}, \eta_{c}^{\mathrm{N}}\right) \quad(\forall a) .
$$

Then the first determinant in (4.40) vanishes because the $b$-th and the $c$-th columns of the matrix are the same. In order for the determinants in (4.40) to be non-vanishing, the components of $\boldsymbol{\eta}^{\mathrm{N}}$ therefore have to be "separated well enough", and similarly for the components of $\boldsymbol{\eta}^{\mathrm{S}}$.

\section{Concluding remarks}

The relation between the gauge theories on vortex defect backgrounds and orbifold backgrounds is useful in studying the physics of the defects using path integral formalism. This approach should be applicable in higher dimensions as well. Similar idea have been employed in the analysis of surface defects in certain $4 \mathrm{D} \mathcal{N}=2$ gauge theories [19].

Our results for non-abelian vortex defects imply there may be a dual description of the defects in terms of local operator insertions. Some defects in higher dimensions are known to have dual "electric" description, namely as low-dimensional field theories on defects interacting with the fields in the bulk $[2,18,29]$. It is interesting to explore other possible descriptions for the point-like vortex defects in $2 \mathrm{D}$ gauge theories.

\section{Acknowledgments}

The author thanks Francesco Benini, Heng-Yu Chen, Kentaro Hori and Tsung-Hsuan Tsai for valuable discussions. He also thanks Yongbin Ruan and Kentaro Hori for the invitation to the workshop on Non-Abelian gauged linear sigma model and geometric representation theory in Beijing where he had useful discussions with the participants. 
Open Access. This article is distributed under the terms of the Creative Commons Attribution License (CC-BY 4.0), which permits any use, distribution and reproduction in any medium, provided the original author(s) and source are credited.

\section{References}

[1] G. 't Hooft, On the Phase Transition Towards Permanent Quark Confinement, Nucl. Phys. B 138 (1978) 1 [INSPIRE].

[2] S. Gukov, Surface Operators, arXiv:1412.7127 [INSPIRE].

[3] C. Kozcaz, S. Pasquetti and N. Wyllard, A $\&$ B model approaches to surface operators and Toda theories, JHEP 08 (2010) 042 [arXiv: 1004.2025] [INSPIRE].

[4] T. Dimofte, S. Gukov and L. Hollands, Vortex Counting and Lagrangian 3-manifolds, Lett. Math. Phys. 98 (2011) 225 [arXiv:1006. 0977] [INSPIRE].

[5] M. Taki, Surface Operator, Bubbling Calabi-Yau and AGT Relation, JHEP 07 (2011) 047 [arXiv: 1007.2524] [INSPIRE].

[6] H. Awata, H. Fuji, H. Kanno, M. Manabe and Y. Yamada, Localization with a Surface Operator, Irregular Conformal Blocks and Open Topological String, Adv. Theor. Math. Phys. 16 (2012) 725 [arXiv: 1008.0574] [INSPIRE].

[7] C. Kozcaz, S. Pasquetti, F. Passerini and N. Wyllard, Affine sl $(N)$ conformal blocks from $\mathcal{N}=2 \mathrm{SU}(N)$ gauge theories, JHEP 01 (2011) 045 [arXiv: 1008.1412] [INSPIRE].

[8] N. Wyllard, W-algebras and surface operators in $N=2$ gauge theories, J. Phys. A 44 (2011) 155401 [arXiv: 1011.0289] [INSPIRE].

[9] N. Wyllard, Instanton partition functions in $N=2 \mathrm{SU}(N)$ gauge theories with a general surface operator and their W-algebra duals, JHEP 02 (2011) 114 [arXiv:1012.1355] [INSPIRE].

[10] G. Bonelli, A. Tanzini and J. Zhao, Vertices, Vortices and Interacting Surface Operators, JHEP 06 (2012) 178 [arXiv:1102.0184] [INSPIRE].

[11] H. Kanno and Y. Tachikawa, Instanton counting with a surface operator and the chain-saw quiver, JHEP 06 (2011) 119 [arXiv:1105.0357] [INSPIRE].

[12] G. Bonelli, A. Tanzini and J. Zhao, The Liouville side of the Vortex, JHEP 09 (2011) 096 [arXiv:1107.2787] [INSPIRE].

[13] Y. Nakayama, $4 D$ and $2 D$ superconformal index with surface operator, JHEP 08 (2011) 084 [arXiv: 1105.4883] [INSPIRE].

[14] D. Gaiotto, L. Rastelli and S.S. Razamat, Bootstrapping the superconformal index with surface defects, JHEP 01 (2013) 022 [arXiv: 1207.3577] [INSPIRE].

[15] L.F. Alday, M. Bullimore, M. Fluder and L. Hollands, Surface defects, the superconformal index and q-deformed Yang-Mills, JHEP 10 (2013) 018 [arXiv:1303.4460] [INSPIRE].

[16] A. Gadde and S. Gukov, 2d Index and Surface operators, JHEP 03 (2014) 080 [arXiv: 1305.0266] [INSPIRE].

[17] M. Bullimore, H.-C. Kim and P. Koroteev, Defects and Quantum Seiberg-Witten Geometry, JHEP 05 (2015) 095 [arXiv: 1412.6081] [INSPIRE].

[18] J. Gomis and B. Le Floch, M2-brane surface operators and gauge theory dualities in Toda, arXiv:1407.1852 [INSPIRE]. 
[19] S. Nawata, Givental J-functions, Quantum integrable systems, AGT relation with surface operator, arXiv:1408.4132 [INSPIRE].

[20] D. Gaiotto and H.-C. Kim, Surface defects and instanton partition functions, arXiv:1412.2781 [INSPIRE].

[21] H.-Y. Chen and T.-H. Tsai, On Higgs branch localization of Seiberg-Witten theories on an ellipsoid, PTEP 2016 (2016) 013B09 [arXiv: 1506.04390] [INSPIRE].

[22] L.F. Alday, D. Gaiotto, S. Gukov, Y. Tachikawa and H. Verlinde, Loop and surface operators in $N=2$ gauge theory and Liouville modular geometry, JHEP 01 (2010) 113 [arXiv:0909.0945] [INSPIRE].

[23] N. Drukker, J. Gomis, T. Okuda and J. Teschner, Gauge Theory Loop Operators and Liouville Theory, JHEP 02 (2010) 057 [arXiv:0909.1105] [INSPIRE].

[24] E. Frenkel, S. Gukov and J. Teschner, Surface Operators and Separation of Variables, JHEP 01 (2016) 179 [arXiv:1506.07508] [INSPIRE].

[25] J. Gomis and B. Le Floch, 't Hooft Operators in Gauge Theory from Toda CFT, JHEP 11 (2011) 114 [arXiv:1008.4139] [INSPIRE].

[26] J. Gomis, T. Okuda and V. Pestun, Exact Results for 't Hooft Loops in Gauge Theories on $S^{4}$, JHEP 05 (2012) 141 [arXiv: 1105.2568] [INSPIRE].

[27] D. Gang, E. Koh and K. Lee, Line Operator Index on $S^{1} \times S^{3}$, JHEP 05 (2012) 007 [arXiv: 1201.5539] [INSPIRE].

[28] T. Okuda, Line operators in supersymmetric gauge theories and the $2 d-4 d$ relation, arXiv:1412.7126 [INSPIRE].

[29] B. Assel and J. Gomis, Mirror Symmetry And Loop Operators, JHEP 11 (2015) 055 [arXiv: 1506.01718] [INSPIRE].

[30] Y. Ito, T. Okuda and M. Taki, Line operators on $S^{1} \times R^{3}$ and quantization of the Hitchin moduli space, JHEP 04 (2012) 010 [arXiv:1111.4221] [INSPIRE].

[31] A. Kapustin, B. Willett and I. Yaakov, Exact results for supersymmetric abelian vortex loops in 2+1 dimensions, JHEP 06 (2013) 099 [arXiv: 1211.2861] [INSPIRE].

[32] N. Drukker, T. Okuda and F. Passerini, Exact results for vortex loop operators in $3 d$ supersymmetric theories, JHEP 07 (2014) 137 [arXiv:1211.3409] [INSPIRE].

[33] I. Biswas, Parabolic bundles as orbifold bundles, Duke Math. J. 88 (1997) 305.

[34] F. Benini and S. Cremonesi, Partition Functions of $\mathcal{N}=(2,2)$ Gauge Theories on $S^{2}$ and Vortices, Commun. Math. Phys. 334 (2015) 1483 [arXiv:1206.2356] [InSPIRE].

[35] N. Doroud, J. Gomis, B. Le Floch and S. Lee, Exact Results in D $=2$ Supersymmetric Gauge Theories, JHEP 05 (2013) 093 [arXiv:1206.2606] [INSPIRE].

[36] J. Gomis and S. Lee, Exact Kähler Potential from Gauge Theory and Mirror Symmetry, JHEP 04 (2013) 019 [arXiv: 1210.6022] [INSPIRE].

[37] T.T. Wu and C.N. Yang, Dirac Monopole Without Strings: Monopole Harmonics, Nucl. Phys. B 107 (1976) 365 [inSPIRE].

[38] V. Pestun, Localization of gauge theory on a four-sphere and supersymmetric Wilson loops, Commun. Math. Phys. 313 (2012) 71 [arXiv:0712. 2824] [InSPIRE]. 